\title{
Long-range attraction between probe particles mediated by a driven fluid
}

\author{
E. Levine ${ }^{(a)}$, D. Mukamel ${ }^{(a)}$, and G.M. Schütz ${ }^{(b)}$ \\ (a) Department of Physics of Complex Systems, Weizmann Institute of Science, Rehovot, Israel 76100. \\ (b)Institut für Festkörperforschung, Forschungszentrum Jülich, 52425 Jülich, Germany.
}

(Dated: April 3, 2018)

\begin{abstract}
The effective interaction between two probe particles in a one-dimensional driven system is studied. The analysis is carried out using an asymmetric simple exclusion process with nearest-neighbor interactions. It is found that the driven fluid mediates an effective long-range attraction between the two probes, with a force that decays at large distances $x$ as $-b / x$, where $b$ is a function of the interaction parameters. Depending on the amplitude $b$ the two probes may form one of three states: (a) an unbound state, where the distance grows diffusively with time; (b) a weakly bound state, in which the distance grows sub-diffusively; and (c) a strongly bound state, where the average distance stays finite in the long time limit. Similar results are found for the behavior of any finite number of probes.

PACS numbers: 02.50.Ey, 05.40.-a
\end{abstract}

Probe particles are a powerful tool in the study of properties of solutions. Interactions between solute particle arise from direct interactions as well as a solventmediated part to which the free energy of solvation contributes. The latter contribution is sometimes rather significant, with surprising effects such as the hydrophobic attraction [1] in which entropy plays the dominant role. Strong entropic effects have also been observed in anisotropic fluids 22]. In equilibrium one frequently approximates these interactions by setting up a suitable model and calculating an effective potential of mean force between solute particles, using the potential distribution theorem [3]. While more quantitative studies require quite sophisticated modelling, simple lattice models often suffice to successfully explain generic features of these interactions. Difficulties of conceptual rather than practical nature, however, arise in equilibrium-based approximation schemes if the solvent is in a strongly nonequilibrium state and therefore no notion of free energy exists. Analyzing the interactions between probe particles in non-equilibrium fluids would thus be of great interest.

Far from equilibrium driven systems of particles moving steadily have been a subject of extensive studies in recent years [4]. The minimal model which has been used to describe these systems is the asymmetric simple exclusion process, whereby self-avoiding particles hop on a lattice with rates which favor motion along the direction of the drive [ 5, [6] . In one dimension (1d) these studies resulted in detailed calculations of a variety of steady state properties, including phase diagrams, density correlation functions and other collective features. In particular, it has been observed that a probe particle introduced into a $1 \mathrm{~d}$ driven fluid is attracted to regions of large density gradient (on molecular scale) in the steady state density profile, thus serving as a microscopic marker of shocks [7]. A local shock is accompanied by a long-range algebraicallydecaying density profile away from the probe. When the system contains two probe particles, the density profile induces a long-range attractive interaction between the probes. For example, in the case of the totally asymmetric simple exclusion process (TASEP) on a ring with two 'second-class' probe particles, the steady-state distribution of the distance $x$ between the two probes was found to decay as $x^{-3 / 2}$ for large $x$ [8]. This implies that the two probes form a weakly bound state, where the average distance is infinite. This exactly soluble case is very specific though, as the second-class particles do not influence the motion of the fluid particles. On the other hand, it is known that the direct interaction between solute and solvent could have a strong dynamical effects, and should not in general be ignored. For example, direct interaction between solute and solvent may lead to a non-monotonic dependence of solute and solvent diffusion coefficients on the solute diameter [9].

In this Letter we present a dynamical approach to the problem of probe particles in a $1 \mathrm{~d}$ driven fluid. This approach is applicable to a broad class of $1 \mathrm{~d}$ systems, which in addition to excluded volume display short range interactions, and where probes directly influence the motion of the fluid particles. Moreover, it enables us to study not only the steady state properties, but also the temporal relaxation to the steady state. We find that within this broader class of systems, two probe particles may form one of three states: unbound, weakly bound, or a strongly bound state. In the unbound state the average distance between the probe particles grows diffusively as $\sqrt{t}$, as is the case for non-interacting particles. In the weakly bound state, the steady-state distance distribution decays algebraically for large $x$ with a power-law $x^{-\sigma}$, where the exponent $1<\sigma<2$ is a function of the interaction parameters. The steady state average distance is infinite. The approach to steady state is sub-diffusive, whereby the average distance grows as $t^{\nu}$ with $\nu<1 / 2$. In the strongly bound state the average distance decays algebraically in time to a finite value at steady state. In 
the steady-state, the distance distribution takes the form $x^{-\sigma}$, with $\sigma>2$. The case of more than two probe particles may also be studied within the approach suggested in this Letter.

We now introduce a model within which the interaction between probes may be analyzed. The model is defined on a 1d lattice of ring geometry, where each site can be occupied by either a positive particle $(+)$, a negative particle (-), or it may be occupied by a probe particle (0). Apart from the exclusion interaction, the 'charged' particles are subject to a nearest-neighbor interaction, defined by the potential

$$
V=-\frac{\epsilon}{4} \sum_{i} s_{i} s_{i+1}
$$

where $s_{i}=0, \pm 1$ according to the occupation of site $i$, and $-0.8<\epsilon<1$ is the coupling constant 10. The dynamics of the model is defined by a random-sequential local dynamics, whereby a pair of nearest-neighbor sites is selected at random, and the particles are exchanged with rates

$$
\begin{array}{lrl}
+- & \rightarrow-+ \\
+0 & \rightarrow 0+, 0-\rightarrow-0 & \text { with rate } 1+\Delta V \\
& \text { with rate } 1 .
\end{array}
$$

Here $\Delta V$ is the difference in the potential $V$ between the initial and final states. This dynamics conserves the number of particles of each species, and is symmetric under the exchange of charges and spatial direction. The model is a generalization of [1] , which has been studied in 12, 13. In the case of no nearest neighbor interaction, $\epsilon=0$, the dynamics defined above reduces to that of the TASEP with second-class particles [7, 8] which are the probes.

In the case of no probe particles, the steady state of this model has an Ising-like measure [11]. This is also expected to be the local steady-state measure away from any probe when the density of probes is zero. We consider first the case of two probe particles in the system. Our aim is to derive dynamical equations for the two probes, and study the evolution in time of the distance $n$ between them, starting from $n=0$.

For $\epsilon=0$ it has been shown [8] that the statistical weight of all configurations with a given $n$ is proportional to $Z_{n}$, the partition function of the TASEP on an open chain of length $n$ in the maximal current phase [5]. This observation has been used to show that the probability to find the two probes at a distance $n$ from each other decays for large $n$ as $n^{-3 / 2}[8]$. In addition, this result can be used to estimate the currents of particles which go in and out of the segment trapped between the two probe particles. One finds that the outgoing current of $+(-)$ particles through the right (left) probe takes the form of the steady state current of the TASEP, given for large $n$ by $j_{n}^{\text {out }}=\frac{1}{4}\left(1+\frac{3 / 2}{n}\right)$. The opposing currents, namely

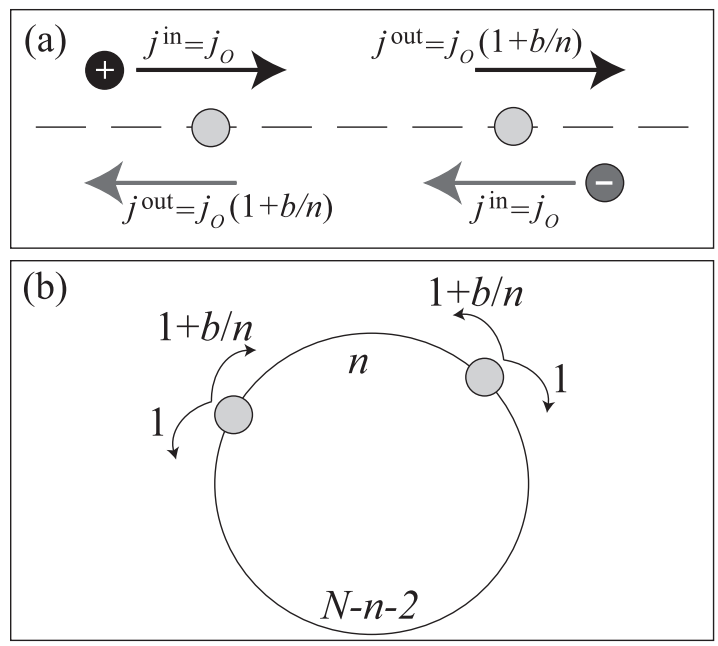

FIG. 1: (a) Schematic diagram of the positive (black) and negative (gray) currents, as seen from the two probe particles. Here $n$ is the distance between the two probes. For $\epsilon=0$ one has $j_{\circ}=1 / 4$ and $b=3 / 2$. (b) A random-walk representation of the dynamics of the two probe particles, as deduced from the currents in (a). Each probe carries out forward and backward hops with the indicated rates.

that of $-(+)$ particles incoming through the right (left) probe, take the form $j^{\text {in }}=\frac{1}{4}\left(1+\frac{3 / 2}{N-n-2}\right)$. In the limit we are concerned with, namely $N \gg n$ and $N \rightarrow \infty$, this current is well approximated by $j^{\text {in }}=\frac{1}{4}$ [Fig. [(a)].

These considerations have been extended to the case $\epsilon \neq 0[12]$. Following $[12]$ one expects that the current of $+(-)$ particles bypassing the right (left) probe takes the same form as that of the current in an open segment of the same length, governed by the same dynamics. This current is given by $j_{n}^{\text {out }}=j_{\circ}(\epsilon)(1+b(\epsilon) / n)$, where

$$
j_{\circ}(\epsilon)=\frac{v+\epsilon}{v^{3}}, \quad b(\epsilon)=\frac{3}{2} \frac{(2+\epsilon) v+2 \epsilon}{2(v+\epsilon)},
$$

and $v=\sqrt{\frac{1+\epsilon}{1-\epsilon}}+1$. For the relevant values of $\epsilon$ one has $0 \leq b \leq 9 / 4$. Also, similar to the $\epsilon=0$ case, the incoming current is given in the large $N$ limit by $j^{\text {in }}=j_{\circ}(\epsilon)$.

The considerations described above may be used to derive dynamical equations for the two probes. These considerations suggest that the two probe particles behave as two coupled random walkers. Each probe moves away from the other probe with rate $j_{\circ}$, and move towards it with rate $j_{\circ}(1+b / n)$ [Fig. प(b)]. Thus, one can model the time evolution of the distance $n$ between the probes by the master equation

$$
\begin{aligned}
& \frac{\partial P(n, t)}{\partial t}=P(n-1, t)-P(n, t) \\
& \quad+\left(1+\frac{b}{n+1}\right) P(n+1, t)-\left(1+\frac{b}{n}\right) P(n, t)
\end{aligned}
$$


for $n>0$, and

$$
\frac{\partial P(0, t)}{\partial t}=-P(0, t)+(1+b) P(1, t)
$$

for $n=0$. Here the time $t$ is a rescaled by a factor $2 j_{0}$. For large $n$ one can use the continuum limit, and (4) can be rewritten as a Fokker-Planck equation (FPE)

$$
\frac{\partial P(x, t)}{\partial t}=\frac{\partial^{2} P(x, t)}{\partial x^{2}}+\frac{\partial}{\partial x}\left(\frac{b}{x} P(x, t)\right),
$$

with the boundary conditions $P(x, t) \rightarrow 0$ as $x \rightarrow \infty$. It is easy to see that the steady-state solution of this equation is of the form

$$
P_{\mathrm{St}}(x) \sim x^{-b} .
$$

This in agreement with the exact result $x^{-3 / 2}$ in the case $\epsilon=0[8]$. Note that the force term $b / x$ in the FPE (5) corresponds to a logarithmically increasing fluid-mediated effective potential $U \sim \ln x$ between the probe particles.

In order to study the approach to steady state we make the scaling hypothesis $P(x, t)=x^{-b} t^{-\beta} f\left(x / t^{\alpha}\right)$, with $f(u)$ a scaling function, which satisfies the boundary condition $f(0)=$ const., and the normalization condition $\int_{\theta}^{\infty} P(x) \mathrm{d} x=1$. Here a cutoff $\theta$ is introduced to prevent divergence at $x \rightarrow 0$ for $b \geq 1$. Substituting this form into (5) one finds that a non-trivial solution exists only when $\alpha=1 / 2$. In the range $b>1$ the normalization condition yields $\beta=0$. Defining $u=x / \sqrt{t}$, the scaling function $f(u)$ satisfies for $b>1$

$$
\left(\frac{1}{2} u^{2}-b\right) f^{\prime}(u)+u f^{\prime \prime}(u)=0 .
$$

This equation reduces to $f^{\prime}(u) \sim u^{b} \exp \left(-u^{2} / 4\right)$, and its solution is given by $f(u) \sim \gamma\left(\frac{b+1}{2}, \frac{u^{2}}{4}\right)$, where $\gamma(a, x)$ is the incomplete $\Gamma$-function. In the range $b<1$ the normalization condition yields $\beta=(1-b) / 2$ and hence

$$
\left(\frac{1}{2} u^{2}-b\right) f^{\prime}(u)+u f^{\prime \prime}(u)=-\frac{1-b}{2} u f(u)
$$

which is solved by $f(u) \sim \exp \left(-u^{2} / 4\right)$.

We now turn to calculate the average distance between the particles, $\langle x(t)\rangle=\int_{\theta}^{\infty} \mathrm{d} x x P(x, t)$. Inspecting the large $t$ behavior of this integral one finds

$$
\langle x(t)\rangle \sim \begin{cases}t^{1 / 2} & b<1 \\ t^{1 / 2} / \log (t) & b=1 \\ t^{1-b / 2} & 1<b<2 . \\ \log (t) & b=2 \\ A+B t^{-(b / 2-1)} & b>2\end{cases}
$$

Here $A$ and $B$ are some non-universal constants. It is evident that depending on the value of $b$ three regimes can

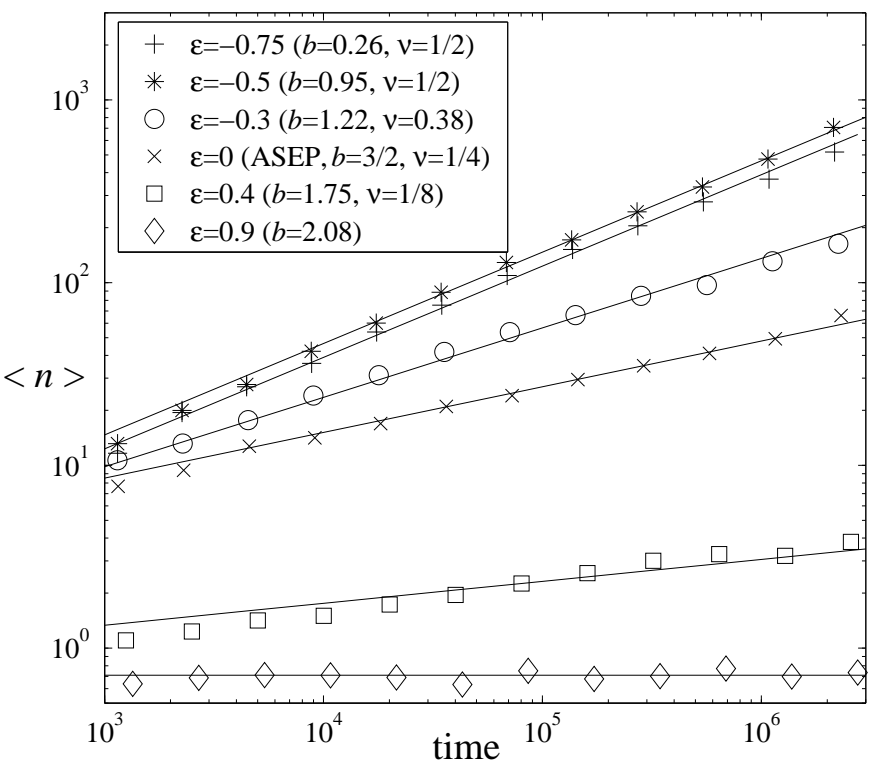

FIG. 2: Results of numerical simulations for the average distance between the two probes for several values of $\epsilon$, and thus of $b$. Lines are drawn with the expected slope, according to Eq. 9

be identified. For $b<1$ the two probes behave as two decoupled particles, and the distance between them grows diffusively. For $1 \leq b \leq 2$ the probes are weakly bound, and the distance between them diverges sub-diffusively. For $b>2$ the two probes are strongly bound, and the average distance between them approaches a constant algebraically in time.

To test the validity of these results we carried out numerical simulations of the model with two probe particles. The simulations were performed on a ring of length $N=10^{5}$, with equal number of + and - particles. Starting with the two probes located on nearest neighbor sites, we followed the evolution of their distance $n(t)$. The average distance $\langle n(t)\rangle$ was obtained by averaging over 5000 realizations of the noise. Results for different values of $b$ in the three regimes are presented in Fig. 20 These results agree very well with the theoretical predictions.

We now consider the case of a finite number $M$ of probes, and show that the scaling behavior, Eq. 9 holds for the average distance between any pair of probes. The evolution of $M$ probes can be be modelled as a zerorange process (ZRP) 14], defined on a one-dimensional ring of $M$ sites, where each site can be occupied by any number of particles. At any time step a site is chosen at random, and one of its particles hops to either one of its two nearest neighbors with the same rate $w_{n}=$ $j_{\circ}(1+b / n)$. Here $n$ is the occupation of the departure site. To make the correspondence between this ZRP and the driven model (2) we identify the occupation number of site $i$ with the distance between the probes $i$ and $i+1$ in the driven model. Moreover, since we are interested 
in the limit $N \rightarrow \infty$, we take site $i=M$ to be occupied by an infinite number of particles. This site serves as a reservoir, omitting particles with current $j_{\text {。 }}$ to both sides. For this zero-range process it can be shown 15] that the distribution of occupation numbers in the steady state takes the form of a product measure,

$$
P_{\text {st }}\left(n_{1}, n_{2}, \cdots, n_{M-1}\right) \sim \prod_{i=1}^{M-1} n_{i}^{-b} .
$$

In the particle system, this steady-state distribution holds exactly only in the case $\epsilon=0$. However, it has been shown that the correlations between adjacent segments can be ignored at large distances $n_{i}$ also for $\epsilon \neq 0$ [12, 13]. With the steady-state distribution at hand, the temporal approach to steady-state can be studied in a similar manner to the case of two probes. In particular, we make the scaling hypothesis

$$
\begin{aligned}
& P\left(x_{1}, \cdots, x_{M-1}, t\right)= \\
& \quad x_{1}^{-b} \cdots x_{M-1}^{-b} t^{-(M-1) \beta} f\left(\frac{x_{1}}{\sqrt{t}}, \cdots, \frac{x_{M-1}}{\sqrt{t}}\right),
\end{aligned}
$$

where, again, $x_{i}$ is the continuous variable corresponding to $n_{i}$. A straightforward analysis of this scaling form shows that (9) holds for all $\left\langle x_{i}\right\rangle$, and thus for the distance between any pair of probes. This agrees well with numerical simulations of systems with 5 probes, supporting the scaling hypothesis (details are not presented).

Our discussion so far was limited to the case where the number of positive particles was equal the number of negative particles. In 13 it has been argued that the physical picture of domain currents underlying the dynamical approach developed above still holds when the densities of the two species are not equal. In this case, the amplitude $b$ becomes a function of both $\epsilon$ and $\eta$, where $\eta$ is the density of, say, the positive particles (see [13] for explicit expressions). It is evident that $b(\epsilon, \eta)$ increases when $\eta$ deviates from $\frac{1}{2}$. Thus, increasing the density of one species at the expense of the other results in larger $b$, and may change the state of the probes from unbound to weakly bound to strongly bound state.

In summary, we introduced in this Letter a dynamical approach for the study of probe particles in onedimensional driven fluids. This approach generalizes the exact result of [8], obtained for the steady-state distribution of two probe particles which do not affect the motion of the fluid, to a broader class of models, which are not exactly solvable. Here any finite number of probe particles, which influence the motion of the fluid, are considered. Moreover, the dynamical approach enables one to study not only steady-state properties, but also the long-time temporal behavior of the probes. It is found that a logarithmically increasing fluid-mediated effective potential $U \sim \ln x$ acts between the probes when they are a distance $x$ apart. It is remarkable that the probe particles behave as if they were in thermal equilibrium even though the fluid is very far from equilibrium. This is reminiscent of the motion of a boundary-induced shock in non-conserving driven systems in contact with a reservoir. The position of the shock has recently been found to perform Brownian motion in an effective potential dynamically generated by the fluid medium [16].

G.M.S. thanks the Weizmann Institute for kind hospitality. The support of the Albert Einstein Minerva Center for Theoretical Physics, Israel Science Foundation, and Deutsche Forschungsgemeinschaft (grant Schu827/4), is gratefully acknowledged.

[1] A. Ben-Naim, Hydrophobic Interactions (Plenum Press, New York, 1980)

[2] P. Poulin, V. Cabuil, and D.A. Weitz, Phys. Rev. Lett. 79, 4862 (1997); E.B. Kim, O. Guzman, S. Grollau, N. Abbott, and J.J. de Pablo, J. Chem. Phys. 121, 1949 (2004).

[3] B. Widom, J. Chem. Phys. 39, 2808 (1963).

[4] See D. Mukamel in Soft and Fragile Matter: Nonequilibrium Dynamics, Metastability and Flow, edited by M.E. Cates and M.R. Evans, (Institute of Physics Publishing, Bristol, 2000); G.M. Schütz, J. Phys. A 36, R339 (2003).

[5] B. Derrida, E. Domany, and D. Mukamel, J. Stat. Phys. 69, 667 (1992); B. Derrida, V. Hakim, M.R. Evans, and V. Pasquier J. Phys. A 26, 1493 (1993); G.M. Schütz and E. Domany J. Stat. Phys. 72, 277 (1993).

[6] See B. Derrida, Phys. Rep. 301, 65 (1998); G.M. Schütz in Phase Transitions and Critical Phenomena vol 19, ed. C. Domb and J. Lebowitz (London: Academic, 2001).

[7] P. Ferrari, Ann. Prob. 14, 1277 (1986); A. De Masi, C. Kipnis, E. Presutti and E. Saada, Stoch. Stoch. Rep. 27, 151 (1989); P. Ferrari, C. Kipnis and E. Saada, Ann. Prob. 19, 226 (1991).

[8] B. Derrida, S.A. Janowsky, J.L. Lebowitz, and E.R. Speer, Europhys. Lett. 22, 651 (1993) ; J. Stat. Phys. 73, 813 (1993); B. Derrida, J.L. Lebowitz, and E.R. Speer, J. Stat. Phys. 89, 135 (1997).

[9] D.N. Perera and P. Harrowell, Phys. Rev. Lett. 80, 4446 (1998).

[10] We limit our discussion here to the case $\epsilon>-0.8$ where the current-density relation has a single maximum at density 1/2, cf. C. Godrèche, E. Levine, and D. Mukamel, in preparation.

[11] S. Katz, J.L. Lebowitz, and H. Spohn, J. Stat. Phys. 34, 497 (1984); J.S. Hager, J. Krug, V. Popkov, and G.M. Schütz, Phys. Rev. E 63, 056110 (2001).

[12] Y. Kafri, E. Levine, D. Mukamel, G.M. Schütz, and R.D. Willmann, Phys. Rev. E 68, 035101 (2003)

[13] M.R. Evans, E. Levine, P.K. Mohanty, and D. Mukamel, Euro. Phys. J. B 41, 223 (2004).

[14] See, e.g., F. Spitzer, Advances in Math. 5, 246 (1970); E.D. Andjel, Ann. Probability 10 525, (1982); M.R. Evans, Braz. J. Phys. 30, 42 (2000).

[15] E. Levine, D. Mukamel, and G.M. Schütz, preprint cond-mat/0412129

[16] A. Rakos, M. Paessens, and G.M. Schütz, Phys. Rev. Lett. 91, 238302 (2003). 